\title{
Testing Fermion Actions: Scaling in the Schwinger Model
}

\author{
Nils Christian ${ }^{1}$, Karl Jansen ${ }^{1}$, Kei-ichi Nagai ${ }^{1}$, Beatrix Pollakowski ${ }^{* 1,2}$ \\ ${ }^{1}$ John von Neumann-Institut für Computing NIC, \\ DESY, Platanenallee 6, D-15738 Zeuthen, Germany \\ 2 Institut für Physik, Humboldt Universität zu Berlin, \\ Newtonstrasse 15, D-12489 Berlin, Germany \\ E-mail: \\ Nils.Christian, Karl.Jansen, Keiichi.Nagai, Beatrix.Pollakowski@desy. de
}

\begin{abstract}
We test the scaling behaviour of Wilson, hypercube, maximally twisted mass and overlap fermion actions in dynamical simulations of the 2-dimensional massive Schwinger model. We also present possibilities to simulate overlap fermions dynamically by replacing the exact overlap operator by an approximate version. This approximation is used either as only the guidance Hamiltonian, keeping the exact overlap operator as the accept/reject Hamiltonian or for both, the guidance and accept/reject Hamiltonian in the Hybrid Monte Carlo algorithm. In the latter case we test whether the approximation error can be corrected for by computing the determinant ratio of the exact and the approximate overlap operators stochastically.
\end{abstract}

XXIIIrd International Symposium on Lattice Field Theory

25-30 July 2005

Trinity College, Dublin, Ireland

${ }^{*}$ Speaker. 


\section{Introduction}

Determining the scaling properties of physical quantities computed with different lattice actions as used presently for dynamical fermion simulations is certainly one of the essential steps to perform a controlled continuum limit. Obviously, performing such scaling tests for lattice QCD in four dimensions is (at least) very demanding. Therefore, we have chosen the 2-dimensional, massive Schwinger model [1] as a test laboratory to address the scaling properties of a number of fermion actions for $N_{f}=2$ flavours of dynamical fermions. In particular, we will compare standard Wilson [2], Wilson twisted mass [3, 4] at maximal twist, hypercube [5] and overlap fermions [6] in their approach to the continuum limit.

In order to study the scaling behaviour, we will fix the scaling variable $z \equiv\left(m_{f} \sqrt{\beta}\right)^{2 / 3}$ to $z=0.2,0.4,0.8$ where $m_{f}$ is the fermion mass in lattice units derived from the PCAC relation and $\beta$ is the coupling multiplying the Wilson plaquette gauge action used throughout this work. Denoting by $e$ the physical gauge coupling and by $a$ the lattice spacing, $\beta=1 / a^{2} e^{2}$. At each of the values of $z$ given above, we compute the pseudo scalar mass and follow its behaviour as a function of (decreasing) values of the lattice spacing. Performing finally a continuum limit of our results allows us to compare to analytical predictions that are available from approximations of the massive Schwinger model which cannot be solved exactly. For a discussion of the scalar condensate we refer to ref. [7], where also a more detailed account of our work can be found.

Another important aspect discussed in this contribution is a description of several ways to perform overlap simulations dynamically. We follow the general idea to replace the exact overlap operator $D_{\mathrm{ov}}$ by some approximate version $D_{\mathrm{ov}}^{\text {approx }}$ having an infra-red safe kernel. To correct for such an approximation we test to reweight by the determinant ratio $\operatorname{det}\left[D_{\mathrm{ov}}\right] / \operatorname{det}\left[D_{\mathrm{ov}}^{\mathrm{approx}}\right]$. We also have tested the possibility to use $D_{\mathrm{ov}}^{\text {approx }}$ as the guidance Hamiltonian in the molecular dynamics part of the Hybrid Monte Carlo (HMC) algorithm while keeping the exact overlap operator $D_{\mathrm{ov}}$ as the accept/reject Hamiltonian.

\section{Scaling results}

The pion mass $m_{\pi}$ and the PCAC fermion mass $m_{\mathrm{PCAC}}$ have been computed by standard methods, using the exponential decay of the 2-point function of the pseudo scalar operator $\tilde{\mathbb{P}}$ and computing the PCAC relation, respectively. In table 1 we list the techniques we have used for the simulations and to compute the correlators numerically for the different fermion actions. We just note that for the twisted mass (tm) fermion case we worked at full twist, realized by the vanishing of the PCAC fermion mass calculated from pure Wilson fermions. In this case, the operators used for the correlation functions need to be appropriately rotated [4], of course. We refer to ref. [7] for the simulation details such as the statistics of our runs and the lattice size and for the precise definitions of the different actions and the notation we have employed. In this reference we also discuss the finite size corrections we have performed for the pion masses when necessary.

In fig. 1 we show one example of the continuum extrapolation of $m_{\pi} \sqrt{\beta}$ for the four different actions we have used. We can see that for all the actions there is a nice scaling with $1 / \beta$, i.e. with $a^{2}$. Another observation is that for all four actions the continuum extrapolated values are consistent, in agreement with universality. Given this finding, we decided to perform constraint 


\begin{tabular}{|l||l|l|}
\hline & Wilson / tm / hypercube & overlap \\
\hline \hline simulations & standard HMC & determinant reweighting \\
\hline operators & conjugate gradient solver & $\begin{array}{l}\text { eigenvalues and eigenvectors } \\
\text { conjugate gradient solver }\end{array}$ \\
\hline currents & local / conserved & local \\
\hline pseudo scalar correlator & $\langle\tilde{\mathbb{P}} \tilde{\mathbb{P}}\rangle$ & $\langle\tilde{\mathbb{P}}-\tilde{\mathbb{S}} \tilde{\mathbb{S}}\rangle$ \\
\hline \hline
\end{tabular}

Table 1: The simulation techniques and the methods to compute the observables for the different actions we have used are listed.

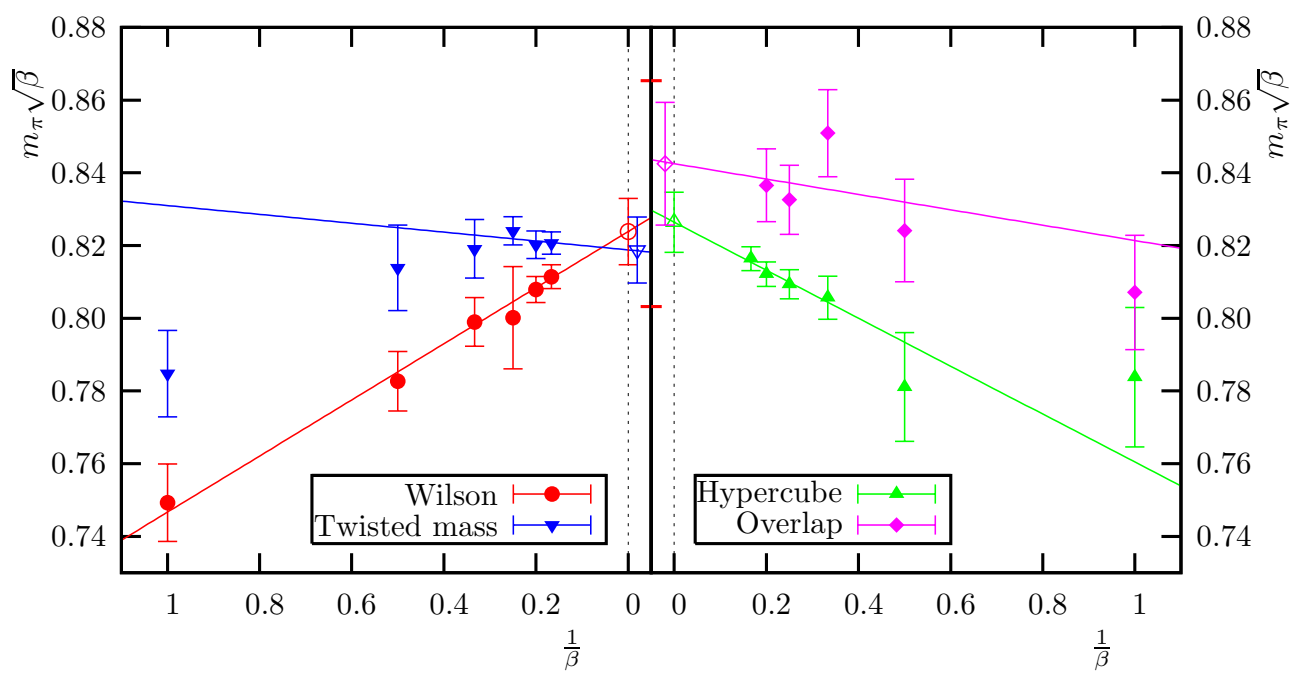

Figure 1: Scaling test of $m_{\pi} \sqrt{\beta}$ as function of $1 / \beta \propto a^{2}$ at fixed value of $z=0.4$.

\begin{tabular}{|l||l|l|l|}
\hline$z=\left(m_{f} \sqrt{\beta}\right)^{2 / 3}$ & $\left(m_{\pi} \sqrt{\beta}\right)_{\mathrm{SG}}$ & $\left(m_{\pi} \sqrt{\beta}\right)_{\mathrm{LG}}$ & $\left(m_{\pi} \sqrt{\beta}\right)_{\mathrm{NP}}$ \\
\hline \hline 0.2 & 0.4016 & 0.43266 & $0.406(4)$ \\
\hline 0.4 & 0.8032 & 0.86532 & $0.825(5)$ \\
\hline 0.8 & 1.6064 & 1.73064 & $1.755(4)$ \\
\hline
\end{tabular}

Table 2: Continuum values for $m_{\pi} \sqrt{\beta}$ at fixed value of $z=\left(m_{f} \sqrt{\beta}\right)^{2 / 3}$. We compare our continuum extrapolated non-perturbatively obtained values, denoted with NP, with theoretical predictions using a SineGordon approach (SG, [8]) and using a large mass approximation (LG, [9]).

continuum extrapolations using fits linear in $1 / \beta$ with a common continuum value for all actions at fixed value of $z$. These final results from our analysis together with theoretical predictions are given in table 2. The theoretical calculations are performed using the Sine-Gordon model [8], denoted as SG in the table, and from a large mass expansion [9] which we denote as LG. Our own non-perturbatively obtained values are denoted as NP. As one can see from table 2, with the high precision we could reach with our simulations, deviations from these theoretical expectations exist. Only for a value of $z=0.2$ there is a consistency with the prediction of ref. [8] while the results of ref. [9] may start to describe the data only for $z=0.8$ or larger. 


\subsection{Simulations}

For the Wilson, the hypercube and the twisted mass fermion action a standard Hybrid Monte Carlo algorithm (HMC) [10] has been implemented for our dynamical simulations. For overlap fermions, we generated configurations with the pure gauge action and then reweighted all observables with the overlap fermion determinant.

The reason for this procedure is that direct simulations of overlap fermions using the HMC algorithm are very difficult and face the generic problem that the kernel operator used in the sign function of the overlap operator can develop very small eigenvalues. We have tried to avoid this problem by replacing $D_{\mathrm{ov}}$ by some operator $D_{\mathrm{ov}}^{\text {approx }}$ that is a good approximation to $D_{\mathrm{ov}}$ but which is safe against these low-lying eigenvalues.

The general idea is to write

$$
\operatorname{det} D_{\mathrm{ov}}=\operatorname{det} D_{\mathrm{ov}}^{\text {approx }} \cdot \operatorname{det}\left[\frac{D_{\mathrm{ov}}}{D_{\mathrm{ov}}^{\text {aprox }}}\right] \equiv \operatorname{det} D_{\mathrm{ov}}^{\text {approx }} \cdot R_{\mathrm{det}} \cdot
$$

One way to implement this idea is to use solely $D_{\mathrm{ov}}^{\text {approx }}$ for the HMC algorithm of the gauge fields. The remaining correction determinant ratio $R_{\text {det }}$ could be implemented as a reweighting factor in the computation of a given observable. Another possibility is to use $D_{\mathrm{ov}}^{\text {approx }}$ as the guidance Hamiltonian in the molecular dynamics part of the HMC algorithm only while the exact overlap operator $D_{\text {ov }}$ is used to evaluate the Hamiltonian for the accept/reject step.

A crucial question in the first of these approaches is, whether an operator $D_{\mathrm{ov}}^{\text {approx }}$ can be found such that the fluctuations in $R_{\text {det }}$ are small enough to obtain statistically significant results. We decided therefore to test this idea by computing $R_{\text {det }}$ on a number of gauge field configurations generated in the pure gauge theory at $\beta=3$ on a $L=16$ lattice.

As approximations to the overlap operator we used the hypercube operator $D_{\text {hyp }}$, an overlap operator with a modified Chebyshev polynomial to evaluate the sign-function and an explicitly infra-red regulated operator, where

$$
D_{0} / \sqrt{D_{0}^{\dagger} D_{0}} \rightarrow D_{0} /\left(\sqrt{\left.D_{0}^{\dagger} D_{0}+\delta\right)},\right.
$$

with $D_{0}$ representing the kernel operator to construct the overlap operator. Out of these set of operators, it turned out that only the operator given in eq. (2.2) led to an acceptable size of the fluctuations for the determinant ratio $R_{\text {det }}$. However, even in this case, when the value of $\delta$ is chosen to be comparable to values of the low-lying eigenvalues of the kernel of the overlap operator, the fluctuations in $R_{\text {det }}$ became so large that this operator does not seem to be practical when the reweighting technique is used, see ref. [7] for a more detailed discussion.

For the hypercube operator it turned out that although the mass parameter was tuned in a wide range, $R_{\text {det }}$ showed always large fluctuations such that no stable result for a physical observable could be obtained. There the square root of the kernel is computed by a Chebyshev polynomial $P_{n, \varepsilon}$ of the degree $n$ in the interval $[\varepsilon, 1]$. In the studies of the modified Chebyshev polynomial $P_{\tilde{n}, \tilde{\varepsilon}}$ we found that it is possible to decrease the degree $n$ of the polynomial to $\tilde{n}<n$, while keeping $\tilde{\varepsilon}=\varepsilon$, by about a factor of three. Although this certainly accelerates the simulations, we also found that already a slight increase of the lower bound $\varepsilon$ of the polynomial approximation to $\tilde{\varepsilon}>\varepsilon$ led again to large fluctuations of $R_{\mathrm{det}}$. 


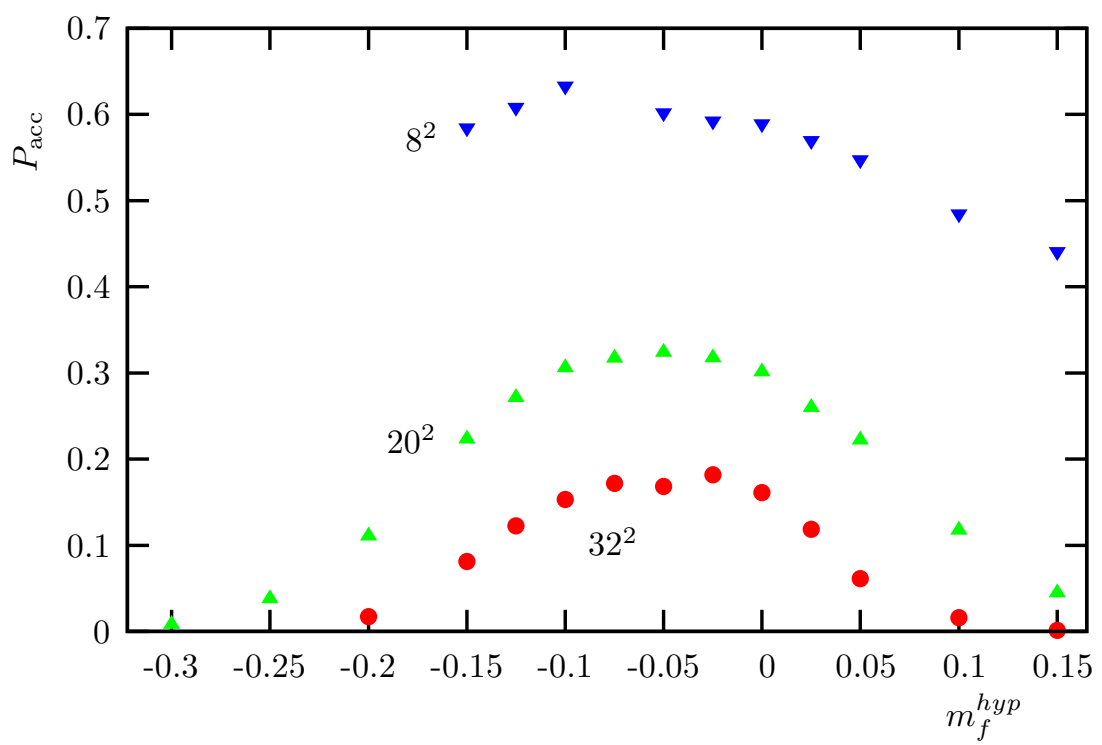

Figure 2: The acceptance rate $P_{\text {acc }}$ using the hypercube operator as the guidance Hamiltonian in the Hybrid Monte Carlo algorithm. $P_{\text {acc }}$ is plotted as a function of the bare overlap fermion mass $m_{f}^{\text {hyp }}$ for three lattice volumes.

As a second attempt, we used an approximate overlap operator for the molecular dynamics part of the HMC algorithm. The setup is then to use $D_{\text {ov }}$ for the accept/reject Hamiltonian while $D_{\text {ov }}^{\text {approx }}$ will be used only for the guidance Hamiltonian. We started to investigate this idea by using the hypercube operator as the guidance Hamiltonian. In fig. 2 we show the acceptance rate as a function of the bare hypercube fermion mass parameter $m_{f}^{\text {hyp }}$ on three lattice volumes. The results are very surprising. From our previous experience with computing $R_{\text {det }}$ we expected a quite bad behaviour of the acceptance rate $P_{\text {acc }}$. In contrast, we find that $P_{\text {acc }}$ is rather large, at least for the $8^{2}$ lattice. Even on our largest lattice $\left(32^{2}\right)$ the acceptance rate does not go to zero but assumes reasonable values. Fig. 2 also demonstrates that $P_{\text {acc }}$ develops a maximum at a certain value of the bare hypercube fermion mass that does not vary strongly with the lattice size. We remark that for a certain threshold step size we could not increase the acceptance rate by decreasing the step size keeping the trajectory length fixed. This means that at this point we are sampling only configurations that are distributed according to the hypercube action.

\section{Conclusions}

In this contribution we have tested four different lattice fermions in their approach to the continuum limit in the 2-dimensional massive Schwinger model with $N_{f}=2$ flavour of dynamical fermions. At fixed scaling variable $z=\left(m_{f} \sqrt{\beta}\right)^{2 / 3}=0.2,0.4,0.8$ we have computed the pseudo scalar mass $m_{\pi} \sqrt{\beta}$ for various values of $\beta=1 / e^{2} a^{2}$.

For all kind of fermions used, Wilson, hypercube, maximally twisted mass and overlap fermions, the scaling behaviour of $m_{\pi} \sqrt{\beta}$ appears to be linear in $a^{2}$ and lead to consistent continuum values, demonstrating nicely universality of the results. The precision of our simulation results reveal that the analytical calculations do not provide a satisfactory description of the data for all values of $z$. 
We also presented possibilities to simulate overlap fermions dynamically by replacing the exact overlap operator by an approximate version which is used either as only the guidance Hamiltonian or as the full Hamiltonian in the Hybrid Monte Carlo algorithm. In the latter case we tested whether the approximation error can be corrected by computing the determinant ratio of the exact and approximate overlap operators stochastically. This way turned out to be not successful since this (correcting) determinant ratio showed large and un-acceptable fluctuations.

Changing the guidance Hamiltonian showed promising results, however. Using the hypercube operator, which led to large fluctuations in evaluating the determinant ratio $R_{\text {det }}$, as the guidance Hamiltonian we find reasonable acceptance rates. Unfortunately, $P_{\text {acc }}$ drops significantly when the lattice volume is increased. If we think of simulations in four dimensions, we therefore have to conclude that the hypercube operator will in this case not be a good choice for the guidance Hamiltonian.

Nevertheless, given the fact that the other approximate overlap operators, modified Chebyshev polynomial and the operator in eq. (2.2), led to much more stable results for the fluctuations of $R_{\text {det }}$, we believe that using these operators as the guidance Hamiltonian should lead to much improved acceptance rates. We are presently testing this idea.

\section{Acknowledgements}

We thank W. Bietenholz, V. Linke, C. Urbach, U. Wenger for many useful discussions. In particular we thank C.B. Lang for pointing out to us to use the modified sign function of eq. (2.2). The computer centers at DESY, Zeuthen, and at the Freie Universität in Berlin provided the necessary technical help and computer resources.

\section{References}

[1] J. S. Schwinger, Gauge invariance and mass. ii, Phys. Rev. 128 (1962) 2425-2429.

[2] K. G. Wilson, Confinement of quarks, Phys. Rev. D10 (1974) 2445-2459.

[3] Alpha Collaboration, R. Frezzotti, P. A. Grassi, S. Sint and P. Weisz, Lattice qcd with a chirally twisted mass term, JHEP 08 (2001) 058 [hep-lat/ 0101001 ].

[4] R. Frezzotti and G. C. Rossi, Chirally improving wilson fermions. i: O(a) improvement, JHEP 08 (2004) 007 [hep-lat/0306014].

[5] P. Hasenfratz, The theoretical background and properties of perfect actions, hep-lat/9803027.

[6] H. Neuberger, Exactly massless quarks on the lattice, Phys. Lett. B417 (1998) 141-144 [hep-lat/9707022].

[7] N. Christian, K. Jansen, K. Nagai and B. Pollakowski, Scaling test of fermion actions in the schwinger model, To be published.

[8] A. V. Smilga, Critical coefficients, Phys. Rev. D55 (1997) 443-447 [hep-th/ 9607154$].$

[9] C. Gattringer, Qed in two-dimensions and u(1) problem, hep-th/9503137.

[10] S. Duane, A. D. Kennedy, B. J. Pendleton and D. Roweth, Hybrid monte carlo, Phys. Lett. B195 (1987) 216-222. 